\title{
ASPECTOS PSICOLÓGICOS EM INDIVÍDUOS COM SOBREPESO E OBESIDADE
}

\section{Isabela Lamante Scotton}

Mestranda pelo Laboratório de Pesquisa e Intervenção Cognitivo-Comportamental, Departamento de Psicologia. Faculdade de Filosofia, Ciências e Letras de Ribeirão Preto - FFCLRP-USP, Brasil

\section{Gabriela Affonso}

Mestre pelo Laboratório de Pesquisa e Intervenção Cognitivo-Comportamental, Departamento de Psicologia. Faculdade de Filosofia, Ciências e Letras de Ribeirão Preto - FFCLRP-USP, Brasil..

\section{Rosane Pilot Pessa}

Livre-docente da Escola de Enfermagem de Ribeirão Preto - EERP-USP, Brasil.

\section{Eva Martins Conceição}

Doutora em Psicologia pela Universidade do Minho, Portugal. Investigadora auxiliar da Escola de Psicologia da Universidade do Minho, Portugal.

\section{Carmem Beatriz Neufeld}

Docente Associada do Departamento de Psicologia da FFCLRP-USP, Brasil.
RESUMO: Avaliar a presença dos sintomas psicológicos em uma amostra de indivíduos com excesso de peso. Estudo transversal com 184 participantes adultos com sobrepeso e obesidade que procuraram atendimento em um programa multidisciplinar de reeducação alimentar. Os instrumentos utilizados foram as escalas de Beck, a de Compulsão Alimentar Periódica e a de Figura de Silhuetas. O nível médio de depressão, ansiedade e desesperança da amostra foi leve, leve e moderado, respectivamente. As mulheres apresentaram maiores níveis em quase todas as variáveis analisadas. Também foram realizadas análises item a item das escalas de Beck e não foram constatadas diferenças em relação ao sexo, com exceção de um item. Podese considerar, portanto, que sintomas de cunho psicológico estão presentes em indivíduos com excesso de peso. Assim, fica clara a importância de considerar os fatores psicológicos na estruturação de intervenções para esta população.

PALAVRAS-CHAVE: Obesidade; Depressão; Ansiedade; Compulsão alimentar.

\section{PSYCHOLOGICAL ASPECTS IN OVERWEIGHT AND OBESE PEOPLE}

\begin{abstract}
To evaluate psychological symptoms in a sample with weight excess. A transversal study with 184 adults featuring overweight and weight, who are attended to in a multidisciplinary program on feed reeducation. Beck's scale, Periodic Feed Compulsion and Silhouette Figure. Mean depression, anxiety and hopelessness of the sample was respectively low, low and moderate. Item by item analyses by Beck's Scale were performed, with no difference with regard to gender, excepting one item. Psychological symptoms exist in overweight people and psychological factors in the structuring of interventions for such a population are highly relevant.
\end{abstract}

KEY WORDS: Obesity; Depression; Anxiety; Food compulsion.

\section{INTRODUÇÃO}

A obesidade é um fenômeno que abrange certa complexidade, pois sua etiologia é multifatorial. Nessa condição, o tratamento farmacológico nem sempre é a primeira opção de tratamento; recomenda-se uma intervenção que seja pautado em uma abordagem multidisciplinar. Apesar de a obesidade ser definida apenas por critérios antropométricos, fatores psicológicos, socioculturais, genéticos e fisiológicos devem ser levados em consideração para se pensar tanto a etiologia 
quanto a manutenção do fenômeno da obesidade ${ }^{4}$. Nesse sentido, o indivíduo com obesidade apresenta aspectos emocionais e psicológicos que podem ser identificados como causadores, mantenedores ou retroalimentadores da obesidade $1,2,3,4$.

Um estudo de revisão5 objetivou analisar a relação entre obesidade e depressão, bem como os fatores psicossociais associados a esta relação. Dentre os resultados, os autores encontraram dados controversos sobre a influência do sexo, sendo que de 20, 12 artigos encontraram que ser mulher confere maior risco de obesidade comórbida com depressão, enquanto oito não encontraram tal relação; os autores também constataram uma relação positiva entre o grau de obesidade e a intensidade dos sintomas depressivos, e que a compulsão alimentar também parece influenciar a relação obesidade-depressão, bem como a imagem corporal, sendo que a superestimação e a insatisfação com o corpo aumentam o risco de uma depressão comórbida.

Outro estudo de revisão ${ }^{6}$ também aponta a relação entre a obesidade e as perturbações do humor, com relações positivas entre o grau de obesidade e a presença/ intensidade dos sintomas depressivos, e que mulheres com excesso de peso possuem maior probabilidade de desenvolver uma perturbação do humor ao longo da vida. As autoras também encontraram que a compulsão alimentar tem influência sobre o desenvolvimento de depressão, nestes indivíduos, porém não encontraram relações significativas entre o grau de compulsão e os valores de IMC.

O mesmo estudo aponta que, apesar dos resultados serem contraditórios, existem evidências moderadas de uma associação positiva entre a obesidade e os transtornos de ansiedade, também relatando diferenças entre os sexos, sendo que mulheres são mais suscetíveis ao desenvolvimento dos sintomas. As autoras também apontam uma relação positiva entre o grau de obesidade e a intensidade da ansiedade, sendo que os indivíduos com maior grau de obesidade apresentam maiores níveis de ansiedade quando comparados com outros níveis.

Os distúrbios alimentares, como a compulsão alimentar, também foram associados ao excesso de peso, à obesidade, à falta de tratamento e ao aumento de peso de pacientes com estas condiçóes. Alguns transtornos alimentares, por um aumento nas calorias consumidas, produzem um equilíbrio calórico positivo que resulta em ganho de peso ${ }^{1}$.

A insatisfação com a imagem corporal também é apontada frequentemente entre os indivíduos com sobrepeso e obesidade, principalmente em mulheres, e está associada com maior incidência de depressão, baixa autoestima e perfeccionismo ${ }^{7,8}$. Estudos mostram que a percepção da imagem corporal afeta o bem-estar psicológico e a habilidade do indivíduo em manter a perda de peso, e que intervenções para perda de peso podem melhorar a imagem corporal nos indivíduos com obesidade?

Os estudos sugerem que as causas de cunho psicológico interferem no desenvolvimento da obesidade, explicitando a importância de se considerar os fatores psicológicos envolvidos no tratamento de perda de peso. Em vista disso, também salienta a necessidade do acompanhamento multidisciplinar a fim de expandir o conhecimento sobre o assunto, pois a diversidade de conhecimentos que estes trazem pode contribuir para a melhor compreensão dos aspectos envolvidos a fim de auxiliar na prevenção e no tratamento do excesso de peso $^{1,10}$.

Considerando-se a importância de um enfoque multidisciplinar para o tratamento da obesidade, a Terapia Cognitivo-Comportamental (TCC) vem demonstrando eficácia por trabalhar os aspectos relacionados ao padrão alimentar disfuncional, como a avaliação e correção de pensamentos inadequados, que contribuem tanto para a etiologia quanto para a manutenção do excesso de peso. A avaliação de tais pensamentos é um procedimento disparador no processo terapêutico para a modificação comportamental, bem como para a reestruturação cognitiva $^{11,12}$. O desafio da TCC recai em compreender como diversos fatores interagem entre si nas situações para favorecer a melhora no manejo do sintoma, de modo que o paciente possa dispor de um repertório qualitativamente mais amplo para lidar com aspectos cotidianos ${ }^{11}$.

É válido destacar que a grande maioria dos estudos sobre aspectos psicológicos associados ao excesso de peso discorrem apenas sobre os aspectos gerais dos sintomas como, por exemplo, escores gerais de depressão e ansiedade. Assim, torna-se importante que o terapeuta em TCC compreenda de forma mais refinada os aspectos 
psicológicos importantes associados ao excesso de peso, como pensamentos e sintomas mais sobressalentes característicos da população a ser atendida por ele.

A partir destes dados, fica clara a necessidade de estudos que se proponham a contribuir para a caracterização psicológica da população com excesso de peso, uma vez que se tratam de fenômenos já prevalentes em nível epidêmico, e com presença em nível global. Sabe-se que a taxa de abandono das intervenções para a redução de peso são elevadas, e que aspectos psicológicos podem comprometer a adesão ao tratamento ${ }^{13}$. Portanto, é essencial que os terapeutas compreendam as características desta clientela. Assim, esse estudo, ao se propor a caracterizar a população que procura esse tipo de tratamento multidisciplinar visa contribuir de forma direta para maior delimitação de uma amostra desta população que procura tratamento, caracterizando-a quanto a importantes indicadores psicológicos. Além disso, contribui para a construção do conhecimento para melhoria da qualidade dos serviços de saúde para essa clientela e o melhor entendimento acerca da etiologia e funcionamento desses fenômenos multifacetados.

O presente estudo tem como objetivo avaliar a presença dos sintomas de ansiedade, depressão, desesperança, acurácia da percepção da imagem corporal e insatisfação com a imagem corporal em uma amostra de indivíduos com excesso de peso que procuraram o atendimento em um programa de educação alimentar em grupo da Universidade de São Paulo, no período de 2010 a 2013.

Além disso, pretende-se investigar, dentre os participantes que apresentarem sintomatologia moderada ou grave de ansiedade, depressão e desesperança, quais são os sintomas mais sobressalentes apontados por eles, a partir da análise item a item dos instrumentos preenchidos, visando uma caracterização mais aprofundada e qualitativamente detalhada em relação aos fatores psicológicos distintivos destes indivíduos.

\section{METODOLOGIA}

Trata-se de um estudo transversal de caracterização de amostra, descritivo e quantitativo. A seguir, o método será detalhado.

\section{CONTEXTO DA PESQUISA}

O presente estudo faz parte de um projeto maior, de educação alimentar (PRAUSP), da Universidade de São Paulo, campus de Ribeirão Preto ${ }^{14}$. O programa conta com uma equipe multidisciplinar composta por alunos e profissionais das áreas de psicologia, nutrição, educação física e enfermagem. A equipe de psicólogos constitui o PRO-META ${ }^{15}$, um programa integrativo do PRAUSP que tem como foco a reestruturação cognitiva dos participantes, trabalhando principalmente as crenças de incapacidade de autocontrole e permissividade que contribuem para a manutenção da obesidade e de hábitos disfuncionais de saúde alimentar.

\section{PARTICIPANTES}

Foram estudados os instrumentos preenchidos por 184 participantes, membros da comunidade USP (funcionários, estudantes e familiares) que procuraram voluntariamente o atendimento no PRAUSP. Os participantes são adultos, entre 18 e 79 anos, com média de idade de 43,36 anos ( $d p=12,38)$, que apresentam sobrepeso e obesidade nos graus I, II e III. Segundo a OMS ${ }^{16}$ uma pessoa com o IMC igual ou superior a $25 \mathrm{Kg} / \mathrm{m}^{2}$ é considerada com sobrepeso, obesidade grau I quando o IMC situa-se 30 e $34,9 \mathrm{~kg} / \mathrm{m}^{2}$, obesidade grau II quando o IMC está entre 35 e $39,9 \mathrm{~kg} / \mathrm{m}^{2}$ e obesidade grau III quando o IMC ultrapassa $40 \mathrm{~kg} / \mathrm{m}^{2}$.Foram analisadas as respostas dos instrumentos dos participantes que realizaram o pré-teste antes do início da intervenção.

\section{PROCEDIMENTO DE COLETA DOS DADOS}

Os participantes realizaram individualmente uma entrevista semiestruturada e os procedimentos de coleta de dados como altura e peso. Estes indivíduos foram submetidos ainda, em grupo, aos questionários clínicos de autoavaliação descritos a seguir, na seção "Instrumentos", excetuando-se a escala de figura de silhuetas que foi aplicada individualmente. Todos os instrumentos foram aplicados antes do programa de intervenção supracitado ter início, bem como a realização da entrevista. 


\section{INSTRUMENTOS}

Escalas de Beck: utilizadas para as avaliações objetivas de humor; foram desenvolvidas por Beck e colaboradores para avaliar aspectos da depressão, ansiedade e desesperança, respectivamente, traduzidos e validados para a população brasileira por Cunha ${ }^{17}$.

Inventário de depressão Beck:

O BDI é uma escala de autorrelato, composto por 21 itens, cada um com quatro alternativas com escores que variam de 0 a 3 que subentendem graus crescentes de depressão (mínimo, leve, moderado e grave). O escore total permite a classificação dos níveis de intensidade da depressão, que variam entre mínimo, leve, moderado e grave $^{18,17}$.

\section{Inventário de ansiedade Beck:}

O BAI também é uma escala de autorrelato, constituído por 21 itens que são "afirmações descritivas de sintomas de ansiedade"18 cada um com quatro alternativas com escores que variam de 0 a 3 , que refletem níveis crescentes de cada sintoma (mínimo, leve, moderado e grave): 0 - "Absolutamente não"; 1 - "Levemente: não me incomodou muito"; 2 - "Moderadamente: foi muito desagradável, mas pude suportar"; 3 - "Gravemente: dificilmente pude suportar". O escore total é o resultado da soma dos escores individuais e permite a classificação dos níveis de intensidade da ansiedade, que variam entre mínimo, leve, moderado e grave ${ }^{19,17}$.

\section{Escala de desesperança Beck:}

A BHS é uma medida da dimensão do pessimismo ou "da extensão das atitudes negativas frente ao futuro" (Beck \& Steer, 1993c). É uma escala dicotômica, que engloba 20 itens que consistem em afirmações que envolvem cognições sobre desesperança, para as quais o indivíduo deve assinalar "certo" ou "errado". O escore total é o resultado da soma dos itens individuais, podendo variar de 0 a 20 pontos, e permitindo a classificação dos níveis de intensidade da desesperança, que podem variar entre mínimo, leve, moderado e grave (14-20) ${ }^{20,17}$.

Escala de compulsão alimentar periódica: ECAP $^{21}$ : esse instrumento consiste em um questionário autoaplicável que avalia a gravidade da compulsão em três medidas: sem compulsão, com compulsão moderada e com compulsão grave. As diferentes afir- mativas são agrupadas em 16 questões e possuem valores que mudam entre $0,1,2$ e 3 . O escore total provém da soma dos escores individuais.

$\underline{\text { Escala de figuras de silhuetas }}^{22}$ : consiste em 30 cartões com silhuetas humanas, sendo 15 de cada sexo. Estas silhuetas foram produzidas a partir de fotos de pessoas reais em diferentes IMC's, que foram transformadas em silhuetas por computação gráfica, apresentadas em cartões plastificados. Para a produção da escala, foi utilizado um intervalo médio de $2,5 \mathrm{~kg} / \mathrm{m} 2$ entre as figuras; portanto, cada figura representa um IMC médio, que consta no verso de cada cartão; os cartões com as silhuetas são dispostos em ordem crescente de IMC diante do participante.

Para avaliação da discrepância da imagem corporal, o participante deve escolher o cartão que ele acredita ser o mais próximo de sua silhueta atual. Assim, a discrepância consiste na comparação entre o IMC do cartão escolhido e o IMC real do indivíduo.

Para avaliação da insatisfação com a imagem corporal, o indivíduo deve apontar, então, qual cartão representa o corpo que ele gostaria de ter. A insatisfação consiste no intervalo entre o IMC do cartão escolhido neste momento com o IMC do cartão apontado como o mais próximo de sua silhueta real.

\section{PROCEDIMENTO DE ANÁLISE DOS DADOS}

Os dados provenientes dos instrumentos foram armazenados em um banco já existente, de acordo com o protocolo do programa, e, a partir dos dados do primeiro semestre de 2013, mais os dados de pré-teste dos grupos anteriores, desde 2010, foi elaborado um novo banco com os dados descritivos da amostra, sendo eles: sexo, idade, escolaridade e IMC, bem como os resultados item a item das escalas de Beck e compulsão alimentar, que foram descritos segundo a média e a frequência. A acurácia e a insatisfação da imagem corporal (dados provenientes da EFS) foram descritos em relação à mediana.

Após a organização do banco, a análise se deu em diferentes etapas: primeiramente foram analisados os dados descritivos da amostra. A seguir, foi investigada a distribuição dos escores gerais dentre os níveis crescentes de intensidade desses indivíduos nos três instrumentos supracitados, bem como a média e desvio-padrão des- 
ses escores. Além disso, foi analisado quantos indivíduos obtiveram escores gerais a partir do nível moderado simultaneamente em mais de um instrumento, bem como seus IMC's médios.

Foi avaliado se havia diferença entre os sexos, entre as classificações de IMC e entre os níveis de escolaridade em relação aos parâmetros já mencionados. Para tanto, os dados foram submetidos, primeiramente, ao teste de normalidade Kolmogorov-Smirnov (Lilliefors). Uma vez verificada a não normalidade da distribuição dos dados optou-se pela utilização de testes não paramétricos.

Dessa forma, utilizou-se o teste estatístico Mann-Whitney para verificar se havia diferença entre os sexos quanto aos parâmetros descritos anteriormente. Também foi utilizado o teste de Kruskal-Wallis para comparação dos mesmos parâmetros, dessa vez em relação às categorias de classificações de IMC, bem como dos níveis de escolaridade.

Só então foi dado início às análises item a item; foram selecionados separadamente os indivíduos que obtiveram escores gerais a partir do nível de intensidade moderado em cada instrumento, e com esses $\mathrm{N}$ foram elaboradas tabelas com a frequência e porcentagem da pontuação em cada alternativa de cada item das três escalas. Finalmente, através do teste do qui-quadrado, verificou-se se houve diferença significativa na pontuação de cada item entre homens e mulheres.

\section{ASPECTOS ÉTICOS}

Esta pesquisa foi aprovada pelo Comitê de Ética em Pesquisa (CAAE: 44971315.3.0000.5407). Os participantes inscreveram-se voluntariamente no PRAUSP, e foi apresentado, antes do início da intervenção, o Termo de Consentimento Informado, Livre e Esclarecido (TCLE), tanto para a participação quanto para o armazenamento dos dados provenientes dos instrumentos de avaliação aplicados no início e no final da intervenção.

Além disso, quando observados sintomas a partir de níveis moderados de depressão ou desesperança, essas pessoas eram chamadas para uma nova entrevista e respondiam ao Inventário de Ideação Suicida de Beck (BSI). A partir disso, se fosse verificada a necessidade, a pessoa era encaminhada para um atendimento individual.

\section{RESULTADOS}

É importante destacar, primeiramente, que para as análises a seguir foram utilizados diferentes valores de $\mathrm{n}$, dado que ao analisar o banco de dados alguns deles estavam faltando, pois os participantes algumas vezes deixaram de responder, ou não eram válidos. Portanto, para cada análise será informado o número da amostra correspondente.

A Tabela 1 demonstra a frequência dos dados sociodemográficos da amostra.

Tabela 1. Distribuição das frequências dos dados sociodemográficos da amostra

\begin{tabular}{|c|c|c|c|}
\hline Variável & Categorias & $\begin{array}{c}\text { N válido } \\
\text { total }\end{array}$ & $\begin{array}{c}\text { Frequência } \\
\%(\mathbf{n})\end{array}$ \\
\hline \multirow{2}{*}{ Sexo } & Mulheres & \multirow{2}{*}{184} & $80,4(148)$ \\
\hline & Homens & & $19,6(36)$ \\
\hline \multirow{6}{*}{$\begin{array}{l}\text { Escolari- } \\
\text { dade }\end{array}$} & $\begin{array}{l}\text { Ensino fundamental } \\
\text { incompleto }\end{array}$ & \multirow{6}{*}{179} & $2,2(4)$ \\
\hline & $\begin{array}{l}\text { Ensino fundamental } \\
\text { completo }\end{array}$ & & $2,8(5)$ \\
\hline & Ensino médio incompleto & & $4,5(8)$ \\
\hline & Ensino médio completo & & $32,4(33)$ \\
\hline & $\begin{array}{l}\text { Ensino superior incom- } \\
\text { pleto }\end{array}$ & & $18,4(52)$ \\
\hline & Ensino superior completo & & $39,7(71)$ \\
\hline \multirow{4}{*}{ IMC } & Sobrepeso & \multirow{4}{*}{173} & $31(54)$ \\
\hline & Obesidade I & & $31(54)$ \\
\hline & Obesidade II & & $23(39)$ \\
\hline & Obesidade III & & $15(26)$ \\
\hline
\end{tabular}

A média de idade $(\mathrm{n}=180)$ total dos participantes foi de 43,3 $(\mathrm{dp}=12,4)$; em relação ao IMC, a média encontrada $(\mathrm{n}=173)$ foi de $33,7 \mathrm{~kg} / \mathrm{m}^{2}(\mathrm{dp}=5,9)$, correspondente à classificação de obesidade grau I.

Com relação aos sintomas psicológicos, a Tabela 2 mostra a distribuição dos escores totais obtidos pelos participantes em cada um dos instrumentos BDI, BAI, BHS e ECAP classificados nos intervalos respectivos de cada instrumento. 
Tabela 2. Distribuição dos escores totais de depressão, ansiedade, desesperança e compulsão alimentar

\begin{tabular}{llcc}
\hline Instrumento & Classificação & N válido & $\begin{array}{c}\text { Frequência } \\
\%(\mathbf{n})\end{array}$ \\
\hline BDI & Mínimo & 169 & $43,2(73)$ \\
& Leve & $37,8(64)$ & \\
& Moderado & $16,5(28)$ & \\
& Grave & $2,4(4)$ & \\
\hline BAI & Mínimo & 173 & $59,3(103)$ \\
& Leve & $23,8(41)$ & \\
& Moderado & $13,4(23)$ & \\
& Grave & $3,5(6)$ & \\
\hline BHS & Mínimo & 180 & $67,8(122)$ \\
& Leve & $21,1(38)$ & \\
& Moderado & $6,7(12)$ & \\
& Grave & $4,4(8)$ & \\
\hline \multirow{2}{*}{ ECAP } & Normal & 184 & $63(116)$ \\
& Variação de inclina- & $26,6(49)$ & \\
& ção ao comer muito & & \\
& CAP & $10,3(19)$ & \\
\hline
\end{tabular}

As Tabelas 3 e 4 apresentam as médias dos escores obtidos nas escalas de Beck, bem como na ECAP e na escala de figuras de silhuetas.

Tabela 3. Médias e classificação dos escores das escalas de Beck e da ECAP

\begin{tabular}{lccc}
\hline Instrumento & N válido & Média (DP) & Classificação \\
\hline BDI & 169 & $13,8(8,4)$ & leve \\
BAI & 173 & $11,1(8,9)$ & leve \\
BHS & 180 & $4,2(3,7)$ & mínimo \\
ECAP & 184 & $16,1(9,2)$ & comer normal \\
\hline
\end{tabular}

Tabela 4. Valores obtidos por meio da escala defFiguras de silhuetas

\begin{tabular}{lcc}
\hline Subescalas da EFS & N válido & Mediana (IQR) \\
\hline Acurácia & 146 & $4,3(5,4)$ \\
Insatisfação & 146 & $-7,5(6,2)$ \\
\hline
\end{tabular}

Como mencionado anteriormente, compararam-se os escores médios obtidos em cada variável em relação ao sexo, aos graus de obesidade e aos níveis de escolaridade.

Em relação ao sexo, o teste estatístico Mann-Whitney, ao comparar os escores médios de cada parâmetro divididos nas categorias de homens e mulheres, apon- tou diferenças em todas as variáveis: IMC $(\mathrm{U}=1,186$; $\mathrm{p}=0)$, BDI $(\mathrm{U}=1749,000 ; \mathrm{p}=0,002), \mathrm{BAI}(\mathrm{U}=1716,000$; $\mathrm{p}=0,001), \quad$ BHS $\quad(\mathrm{U}=1877,500 ; \quad \mathrm{p}=0,006), \quad$ ECAP $(\mathrm{U}=1754,000 ; \mathrm{p}=0,001)$ e acurácia $(\mathrm{U}=1,069 ; \mathrm{p}=0)$, exceto na idade $(U=2,162 ; p=0,330)$ e na insatisfação (U= 1776,500; $\mathrm{p}=0,676)$ (nível de significância $\mathrm{p}<0,05)$. Nas variáveis que apresentaram diferença, as mulheres alcançaram os escores mais altos, com exceção do IMC, em que os homens apresentaram maiores valores.

Em relação ao IMC, o teste de Kruskal-Wallis não apresentou diferenças nas variáveis $\mathrm{BDI}$ geral $\left(\mathrm{X}^{2}(3)=0,536 ; \mathrm{p}=0,911\right)$, BAI geral $\left(\mathrm{X}^{2}(3)=0,363\right.$; $\mathrm{p}=0,948)$, BHS geral $\left(\mathrm{X}^{2}(3)=1,281 ; \mathrm{p}=0,734\right)$ e ECAP $\left(X^{2}(3)=5,910 ; p=0,116\right)$. Entretanto, apresentou diferenças significativas em relação à acurácia $\left(X^{2}(3)=35,013\right.$; $\mathrm{p}=0)$ e à insatisfação $\left(\mathrm{X}^{2}(3)=10,684 ; \mathrm{p}=0,14\right)$ (nível de significância $\mathrm{p}<0,05)$.

No que diz respeito à análise de diferença das médias dos parâmetros em relação aos níveis de escolaridade, sendo BDI geral $\left(\mathrm{X}^{2}(5)=9,951 ; \mathrm{p}=0,077\right)$, BAI geral $\left(X^{2}(5)=5,964 ; p=0,310\right)$, BHS geral $\left(X^{2}(5)=9,373\right.$; $\mathrm{p}=0,095)$ e $\operatorname{ECAP}\left(\mathrm{X}^{2}(5)=3,486 ; \mathrm{p}=0,625\right)$, acurácia $\left(X^{2}(5)=4,464 ; p=0,485\right)$ e à insatisfação $\left(X^{2}(5)=6,805\right.$; $\mathrm{p}=0,263)$ o teste Kruskal-Wallis não apontou diferenças em nenhuma variável (nível de significância $p<0,05$ ).

Com relação às análises item a item, no BDI observa-se o seguinte fenômeno: quando se levou em consideração apenas os indivíduos que obtiveram um escore geral de moderado a grave, nota-se que apenas os itens relacionados à autoacusações, indecisão e retração da imagem corporal foram pontuados pela maioria das pessoas nas alternativas 2 e 3 , que refletem níveis moderado e grave, respectivamente, dos sintomas correspondentes. No restante dos itens a maioria das pessoas respondeu pontuando a alternativa 1 , que corresponde a uma intensidade leve do sintoma. Assim, percebe-se que, mesmo em indivíduos que se classificaram nos escores gerais mais graves, a intensidade da maioria dos sintomas elencados na escala é leve; os escores gerais mais elevados, portanto, se dão em torno desses três sintomas, sendo estes mais representativos dessa amostra.

Já com relação ao BAI, observa-se que nove dos 21 sintomas relacionados foram pontuados pela maioria 
dos indivíduos na intensidade 2 (moderado), sendo eles: sensação de calor, incapaz de relaxar, medo que aconteça o pior, palpitação ou aceleração do coração, nervoso, sensação de sufocação, medo de perder o controle, indigestão ou desconforto no abdômen e suor (não devido ao calor), sendo que nenhum item foi majoritariamente pontuado com intensidade grave (3).

No que diz respeito às análises da BHS, observa-se que 14 itens foram pontuados por mais de 50\% dos indivíduos; já era esperado que essa escala apresentasse a maioria dos itens pontuados, já que estão sendo investigados os instrumentos com escores gerais mais elevados, e sendo esta uma escala dicotômica. Entretanto, é interessante observar que alguns itens foram pontuados por mais de $80 \%$ das pessoas, sendo eles: "4. Não consigo imaginar que espécie de vida será a minha em dez anos", "8. Acontece que tenho uma sorte especial e espero conseguir mais coisas boas na vida do que uma pessoa comum", "10. Minhas experiências passadas me prepararam bem para o futuro", " 14 . As coisas simplesmente não se resolvem da maneira que eu quero" e "18. O futuro me parece vago e incerto".

Como dito anteriormente, comparou-se cada item dos três instrumentos em relação ao sexo dos participantes; o teste qui-quadrado apontou diferença apenas para o item 7 do BDI $\left(X^{2}(3)=157 ; p=0\right)$, item que se refere a autodepreciação, sendo que as mulheres foram as que mais pontuaram esse item. Nenhum item do BAI apontou diferenças entre homens e mulheres, e, na BHS, apenas mulheres pontuaram escores gerais de moderado a grave, portanto não foi possível realizar essa análise.

\section{DISCUSSÃO}

A partir dos resultados observados, pode-se observar que a maioria dos participantes do presente estudo $(80,4) \%$ são mulheres, o que é compatível com dados da literatura de que mais mulheres do que homens procuram tratamento para o sobrepeso e a obesidade. A proporção de mulheres é, inclusive, semelhante à proporção encontrada em diversos estudos, como o de Matos e colaboradores ${ }^{23}$ e o de Soares ${ }^{24}$, com porcentagens próximas ou superiores a $80 \%$. Além disso, outros estudos foram realizados apenas com mulheres, pela conveniência da amostra ${ }^{25,26}$.

Esses dados abrem questões para as causas desse fenômeno, que segundo Oliveira e Silva ${ }^{27}$, pode ocorrer pelo fato de que mulheres buscam mais a melhor aparência do que os homens e em casos de problemas de saúde são mais propensas a procurarem tratamento. Já Porto e colaboradores $^{28}$ colocam que pode ser pela procura ter mais mulheres com atividades domésticas, o que as dá mais tempo para procurarem o tratamento. Um ponto importante a ser observado é que homens e mulheres diferem nesta amostra, em relação ao IMC, sendo que a média do IMC dos homens é mais alta $38,2 \mathrm{~kg} / \mathrm{m}^{2}$, enquanto a das mulheres é de $32,5 \mathrm{~kg} / \mathrm{m}^{2}$. Sabendo-se da alta correlação entre obesidade e sintomas psicológicos encontrada pelos estudos, fazem-se necessárias políticas de incentivo aos homens obesos a procurarem também este tipo tratamento, visando promover o bem-estar destes indivíduos, tanto físico quanto psicológico.

A média de idade dos participantes foi também semelhante a outros estudos com indivíduos obesos ${ }^{29}$, sugerindo que a preocupação com a questão do peso ocorre tipicamente na faixa dos 40 anos, por esta população. Entretanto, nota-se que o desvio-padrão é bem alto $(12,4)$, refletindo que procuram pelo serviço do PRO-META indivíduos com uma extensão grande de idade, sendo desde estudantes de graduação e pós-graduação até idosos que fazem parte da comunidade e frequentam outros serviços-escola da Universidade. A partir disso, faz-se necessário que as intervenções do PRO-META sejam pensadas para serem adequadas a estas distintas faixas etárias como, por exemplo, adequar a linguagem à postura dos terapeutas, não sendo extremamente formal nem informal, trazer e adaptar exemplos variados ao trabalhar os exercícios nas sessões, visando abranger contextos de pessoas mais jovens e também dos participantes mais idosos, para que todos sintam-se contemplados.

Com relação à escolaridade, observa-se que uma parcela expressiva da amostra (39,7\%) possui ensino superior completo. Isso pode se dever ao fato de que a divulgação do programa acontece apenas para a comunidade USP, atraindo bastantes professores e alunos de pós-graduação. Há também uma porcentagem próxima de indivíduos com ensino médio completo $(34,2 \%)$, segui- 
do de participantes cursando o ensino superior $(18,4 \%)$. Esses indivíduos somam quase a totalidade da amostra, evidenciando que a procura por neste serviço ocorre majoritariamente por indivíduos de alta escolaridade. Não foram observadas diferenças nos parâmetros psicológicos entre os diferentes níveis de escolaridade, corroborando estudos anteriormente realizados ${ }^{29,30}$.

Entretanto, na prática das autoras, nota-se que, apesar de a maioria dos participantes possuírem alta escolaridade, em quase todos os grupos (formados semestralmente) existe uma pequena porcentagem de participantes de escolaridade mais baixa, com apenas o ensino fundamental completo; tipicamente, são participantes que possuem mais dificuldade de compreensão dos conceitos e que muitas vezes acabam por deixar o grupo logo no início ou na metade da intervenção. É necessário, portanto, treinar os terapeutas para não relegarem acidentalmente estes participantes, já que a maioria demonstra compreender o conteúdo das sessões. O coterapeuta, neste momento, torna-se fundamental, pois possui o papel de identificar estes indivíduos com maiores dificuldades. Assim, a equipe pode desenvolver estratégias para diminuir as taxas de evasão dos participantes e, consequentemente, promover melhor aproveitamento de todos, evitando o sentimento de não-pertencimento destes indivíduos. Por exemplo, pode ser oferecido um auxílio individual após o final da sessão sobre a tarefa de casa.

Em relação aos indicadores psicológicos, os escores gerais de depressão, ansiedade e desesperança aparecem na literatura em menor proporção do que nos estudos anteriormente encontrados ${ }^{23,31,32}$, com exceção do estudo de Saueressig e Berleze ${ }^{33}$. Quando levados em consideração níveis de moderado a grave, pode-se observar que, nesta população, 18,93\% apresentam sintomas depressivos, 16,86\% apresentam sintomas de ansiedade e 11,11\% apresentam sintomas de desesperança. Esse número reduzido pode ser pelo fato de os participantes desses outros estudos estarem procurando uma medida mais drástica, como a intervenção cirúrgica para a obesidade, e por isso possuem os IMC's mais elevados; no programa de reeducação alimentar do qual o presente estudo coletou os dados, há uma procura maior por pessoas com sobrepeso e obesidade grau I (somando 62\%), o que pode explicar os índices mais baixos de sintomatologia psico- lógica, dado que outros estudos ${ }^{27,35}$ relatam correlação positiva entre IMC e gravidade da sintomatologia. No que diz respeito à diferença entre os sexos, as três variáveis apresentaram diferenças entre homens e mulheres, sendo que as mulheres apresentam escores médios maiores em todas elas.

A EFS mostrou, por meio da medida da acurácia, que em geral os participantes superestimaram seus corpos, uma vez que a mediana é 4,3 $(\mathrm{IQR}=5,4)$. Quando comparado esse parâmetro em relação aos sexos, percebe-se diferença significativa, sendo que as mulheres tendem a superestimar mais os seus corpos (média 4,7, significando que as mulheres superestimam seus corpos em 4,7 vezes em média a mais do que seu IMC real) do que os homens (média 1,3) $(\mathrm{p}=0)$.

Em relação à insatisfação, o índice foi mais contundente, evidenciando que $100 \%$ das pessoas em maior ou menor grau estão insatisfeitas com a percepção que tem de seus corpos. Uma vez que a mediana foi de -7,5 (IQR= $6,2)$, isso significa que as pessoas em geral gostariam de ter seus corpos bem mais magros. Não foram observadas diferenças entre homens e mulheres $(p=0,67)$, corroborando os dados e Kakeshita \& Almeida ${ }^{22}$.

Levando-se em consideração estes dados da escala de Silhuetas, faz-se necessário repensar a intervenção do PRO-META e de programas semelhantes visando adicionar estratégias específicas voltadas para a percepção da imagem corporal, visto que esta intervenção ainda possui foco na reestruturação cognitva dos indivíduos. Apesar de as mulheres superestimarem mais os seus corpos, observa-se a alta insatisfação com a imagem corporal por todos os participantes, sugerindo que tais estratégias poderiam beneficiar de forma significativa os que procuram o serviço.

Outro dado bastante interessante foi o apontado pelo teste de Kruskal-Wallis em relação a diferenças entre as classificações de IMC. As medidas de acurácia e insatisfação foram os únicos parâmetros nos quais foi sinalizada diferença entre as classificações de IMC. De forma grosseira pode-se observar que as médias da acurácia tendem a diminuir com o aumento do IMC, enquanto os valores da insatisfação tendem a aumentar. Ou seja, conforme aumenta o IMC, as pessoas tenderam a subestimar seu tamanho corporal, percepção esta diferente entre homens e 
mulheres como dito acima. Por outro lado, independente do sexo, com o aumento do IMC, as pessoas tenderam a demonstrar maior insatisfação com seus corpos.

A variável que teve maior impacto na amostra foi a ECAP, cuja porcentagem de sintomas foi 36,9\% (considerados indicadores de sintomas a partir da classificação Variação de inclinação ao comer muito) corroborando com os estudos epidemiológicos que apontam que cerca de $30 \%$ das pessoas que procuram tratamentos para emagrecer tem Transtorno de Compulsão Alimentar Periódica ${ }^{34}$

Também pode ser observada diferença entre homens e mulheres no que diz respeito às médias da ECAP, sendo que as mulheres em média possuem inclinação ao comer muito (média 17,0), enquanto os homens em média não possuem compulsão (média 11,7). Levando em consideração a alta proporção de mulheres na amostra, faz-se necessário investir também em estratégias voltadas para este transtorno. Segundo Duchesne e Almeida ${ }^{34}$, a Terapia Cognitivo-Comportamental apresenta alta eficácia para o tratamento do TCAP, porém estudos mais recentes vêm apontando o crescimento da utilização de estratégias de mindfulness para o transtorno (ref Almeida e Assumpção 2018). Assim, exercícios de mindful eating, por exemplo, podem trazer um benefício significativo para a intervenção do PRO-META e serviços semelhantes. Não houve diferenças na média dos sintomas de ECAP nas diferentes classificações de IMC $(p=0,116)$, sugerindo que os participantes de todas as faixas podem beneficiar-se de tais estratégias.

Com relação às análises item a item, no BDI observa-se o seguinte fenômeno: quando se levou em consideração apenas os indivíduos que obtiveram um escore geral de moderado a grave, nota-se que apenas os itens relacionados à autoacusações, indecisão e retração da imagem corporal foram pontuados pela maioria das pessoas nas alternativas 2 e 3 , que refletem níveis moderado e grave, respectivamente, dos sintomas correspondentes. No restante dos itens a maioria das pessoas respondeu pontuando a alternativa 1 , que corresponde a uma intensidade leve do sintoma. Assim, percebe-se que, mesmo em indivíduos que se classificaram nos escores gerais mais graves, a intensidade da maioria dos sintomas elencados na escala é leve; os escores gerais mais elevados, portanto, se dão em torno desses três sintomas, sendo estes mais representativos dessa amostra.

Foram descritas duas subescalas do BDI: uma com itens cognitivo-afetivos, que são os 13 primeiros itens do inventário, e uma segunda subescala com queixas somáticas, composta pelos oito últimos itens ${ }^{18}$. Os itens referentes às autoacusações e à indecisão integram a primeira subescala, enquanto o item referente à retração da imagem corporal faz parte da segunda. Nota-se, portanto, que ambas as dimensões da sintomatologia da depressão elencadas nessa escala são importantes para pensar essa amostra.

É interessante destacar que a preocupação com a imagem corporal também aparece em outros estudos com indivíduos com obesidade, como o de Matos e colaboradores ${ }^{23}$, que constataram essa característica em $76 \%$ de sua amostra. Este dado reforça a necessidade de pensar em estratégias voltadas para a imagem corporal, já apontada a partir dos resultados da escala de Silhuetas.

Já com relação ao BAI, observa-se que nove dos 21 sintomas relacionados foram pontuados pela maioria dos indivíduos na intensidade 2 (moderado). Assim, pode-se dizer que, nessa amostra, a ansiedade dos indivíduos se dá de forma que os sintomas se apresentam mais difusos na escala, ou seja, mais sintomas são responsáveis pelas condições moderada e grave dos escores gerais nesse instrumento, quando comparados aos sintomas de depressão, apesar de os indivíduos que apresentaram escores gerais mais elevados no BAI constituírem menor porcentagem da amostra total do que no BDI.

É interessante observar que os sintomas mais pontuados são tanto de natureza mais cognitiva (incapaz de relaxar, medo de que aconteça o pior, nervoso e medo de perder o controle) quanto fisiológica (sensação de calor, palpitação ou aceleração do coração, sensação de sufocação, indigestão ou desconforto do abdômen e suor), sendo importante pensar ambas as dimensões da ansiedade ao planejar intervenções para essa população. Sugere-se, portanto, acrescentar estratégias de manejo da emoção à intervenção, como a respiração diafragmática e o relaxamento muscular progressivo ${ }^{35}$, que atua no sistema nervoso autônomo reduzindo sintomas físicos de ansiedade, bem como técnicas cognitivas para o manejo deste transtorno.

Silvestri e Stavile ${ }^{36}$ colocam que uma das causas 
mais frequentes para o aparecimento de uma ansiedade crônica é o medo de controlar os impulsos e de perder o controle dos acontecimentos na vida, fazendo com que uma pontuação alta no item "medo de perder o controle" seja condizente com esse dado. Outras causas colocadas pelos autores diz respeito ao medo de perder a estabilidade, de ser abandonado e da repetição de acontecimentos traumáticos, o que se pode relacionar com o sintoma "medo de que aconteça o pior", também bastante pontuado pela amostra.

Esses autores também colocam que a forma de ansiedade mais frequentemente relacionada com a obesidade é a ansiedade generalizada, o que quer dizer que ela está presente em níveis não muito elevados, porém quase permanente, e que por isso os indivíduos conseguem uma porcentagem importante de redução por meio do ato de comer. Pode-se relacionar esse dado à forma como o instrumento foi pontuado, pois como relatado acima, nenhum item foi majoritamente pontuado no nível 3 (grave), e vários itens foram pontuados com menores intensidades, reforçando esse dado. Assim, ao pensar as intervenções voltadas para a ansiedade nesta amostra, sugere-se que os terapeutas investiguem estratégias cognitivas e comportamentais eficazes para a ansiedade generalizada, como relaxamento, intolerância à incerteza, reestruturação de crenças errôeas sobre preocupação, resolução de problemas, entre outras ${ }^{37}$.

No que diz respeito às análises da BHS, observa-se que 14 itens foram pontuados por mais de $50 \%$ dos indivíduos; já era esperado que essa escala apresentasse a maioria dos itens pontuados, já que estão sendo investigados os instrumentos com escores gerais mais elevados, e sendo esta uma escala dicotômica. Entretanto, é interessante observar que alguns itens foram pontuados por mais de $80 \%$ das pessoas, sendo eles: "4. Não consigo imaginar que espécie de vida será a minha em dez anos", "8. Acontece que tenho uma sorte especial e espero conseguir mais coisas boas na vida do que uma pessoa comum", "10. Minhas experiências passadas me prepararam bem para o futuro", " 14 . As coisas simplesmente não se resolvem da maneira que eu quero" e " 18 . O futuro me parece vago e incerto". Assim, é importante atentar para essas cognições em relação ao futuro ao se pensar essa amostra, podendo valer-se o terapeuta da reestruturação cognitiva.

Com relação à comparação entre cada item dos instrumentos com o sexo dos participantes, é interessante notar que não há diferenças entre homens e mulheres, com exceção do item 7 do BDI, referente à autodepreciação, em que as mulheres pontuaram mais que os homens. Levando em consideração estes dados, é válido destacar que, apesar de as mulheres apresentarem maiores escores gerais de sintomatologia psicológica, ambos os sexos respondem de maneira semelhante a aspectos específicos da depressão e ansiedade (quando levados em consideração indivíduos que possuem sintomas de moderado a grave de depressão e ansiedade). Portanto, apesar de as mulheres procurarem mais o tratamento multidisciplinar para sobrepeso e obesidade, nota-se que os homens possuem praticamente as mesmas preocupações que as mulheres, porém procuram menos ajuda para estes fenômenos. Esse fato reforça ainda mais a necessidade de incentivar a procura dos homens pelo serviço. Para o PRAUSP, isso significa repensar as estratégias de divulgação.

É notável observar, entretanto, que apenas mulheres pontuaram de moderado a grave na BHS, sugerindo que sintomas de desesperança estão presentes na amostra, dada a alta proporção de mulheres. Sugere-se, entretanto, que outros estudos realizem análises semelhantes com amostras mais abrangentes para conclusões mais contundentes.

\section{CONCLUSÃO}

Frente aos dados encontrados e os achados da literatura, pode-se considerar que os sintomas de cunho psicológico estão presentes em indivíduos com sobrepeso e obesidade, em alguma proporção. Dessa forma, observa-se a importância de se considerar os fatores psicológicos envolvidos no tratamento de perda de peso e ponderar esses aspectos na estruturação de uma intervenção mais eficaz para cada perfil de amostra, sabendo que tais aspectos tendem a contribuir com a adesão destes indivíduos ao tratamento.

Portanto, esse estudo objetivou contribuir para melhor caracterização dos aspectos psicológicos dos indivíduos que buscam tratamento, possibilitando um 
manejo mais adequado das mesmas, uma vez que estes fatores interferem na eficácia dos tratamentos de perda de peso, como já foi evidenciado. Considerando as características da TCC para o tratamento deste fenômeno, torna-se importante identificar, dentre os indivíduos com escores elevados de sintomas de depressão, ansiedade e desesperança, quais são os aspectos mais distintivos desta população.

Para que tal contribuição tenha aplicações práticas, é preciso repensar as formas de intervenção psicológica no atendimento a esse público-alvo, assim como desenvolver estratégias para trabalhar todas essas demandas conjuntamente, levando em conta as características do pensamento das pessoas que buscam este tipo de intervenção, que podem ser observadas, dentre outras formas, pelos resultados item a item dos instrumentos. Dado que, no PRO-META, a intervenção em TCC é baseada na reestruturação cognitiva dos participantes em relação aos comportamentos alimentares, este estudo contribui para a reflexão acerca do programa sobre como estruturar as sessões de maneira a abarcar também as particularidades dos participantes com sintomatologia psicológica mais intensificada. Os dados encontrados neste estudo permite direcionamentos específicos para a melhoria do programa PRO-META, como a possibilidade da inserção de estratégias como manejo da ansiedade, reestruturação de cognições de desesperança, exercícios de mindful eating, intervenções específicas para imagem corporal, entre outras.Tais dados também podem contribuir para a estruturação de outros programas que tenham como finalidade o tratamento psicológico de pessoas com excesso de peso.

É válido ressaltar que se tratou de um estudo inicial que permitiu observar como essas variáveis aparecem nessa população específica que procura 0 PRAUSP. Uma limitação do estudo consiste no fato de que a amostra não se apresentou de forma pareada, sendo que aproximadamente $80 \%$ desta é composta por indivíduos do sexo feminino. Sugerem-se estudos que aprofundem as análises de caracterização, buscando investigar outra amostras, por exemplo, e relações entre as variáveis observadas como apresentados em outros estudos, entre IMC e gravidade dos sintomas. Assim, possibilita-se uma análise mais abrangente, para dar subsídios para melhor entendimento acerca do funcionamento desses fenômenos multifacetados e, consequentemente, contribuir na formação de conhecimento para melhoria do atendimento a essa população.

\section{REFERÊNCIAS}

1. Vargas V. Elementos para pensar a obesidade, a depressão e a ansiedade [Trabalho de Conclusão de Curso]. Ijuí: Universidade Regional do Noroeste do Estado do Rio Grande do Sul; 2012.

2. Errandonea UI. Obesidad y trastornos de alimentación. Revista Médica Clínica Las Condes. 2012; 23(2): 165-171.

3. Gordon PC, Kaio, GH, Sallet, PC. Aspectos do acompanhamento psiquiátrico de pacientes obesos sob tratamento bariátrico: Revisão. Revista de Psiquiatria Clinica. 2012; 38(4): 148-154.

4. Henriques S, Cruz D, Basso R, Cleonice Z, Bosa A. Relação entre Apego e Obesidade: Revisão Sistemática da Literatura. Psico. 2015; 46(1): 6-15.

5. Preiss K, Brennan L, Clarke D. A systematic review of variables associated with the relationship between obesity and depression. Obesity Reviews. 2013; 14(11): 906-918.

6. Pereira C, Brandão I. Uma Perspetiva da Psicopatologia da Obesidade. Arquivos de Medicina. 2014; 28(5): 152-159.

7. Brandão I, Fernandes AL, Osório E, Calhau MC, Coelho R. A psychiatric perspective view of bariatric surgery patients. Revista de Psiquiatria Clinica. 2015; 42(5): 122-128.

8. Weinberger NA, Kersting A, Riedel-Heller SG, Luck-Sikorski C. Body Dissatisfaction in Individuals with Obesity Compared to Normal-Weight Individuals: A Systematic Review and Meta-Analysis. Obesity Facts. 2017; 9(6): 424-441.

9. Chao HL. Body image change in obese and overweight persons enrolled in weight loss intervention programs: A systematic review and meta-analysis. PLoS ONE. 2015; 10(5): 1-12.

10. Mendes AA, Ieker ASD, de Castro TF, Avelar A, Nardo Júnior N. Multidisciplinary programs for obesity treatment in Brazil: A systematic review. Revista de 
Nutrição. 2016; 29(6): 867-884.

11. Vasques F, Martins FC, Azevedo AP. Aspectos psiquiátricos do tratamento da obesidade. Revista de Psiquiatria Clínica. 2004; 31(4): 195-198.

12. Lima ACR, Oliveira AB. Fatores psicológicos da obesidade e alguns apontamentos sobre a terapia cognitivo-comportamental. Mudanças-Psicologia da Saúde. 2016; 24(1): 1-14.

13. Palmeira SC, Garrido M, Maria L, Resumo PS. Factores intervinientes en la adhesión al tratamiento de la obesidad. Ciencia y Enfermería. 2016; 22(1): 11-22.

14. Bueno JM, Leal FS, Saquy LPL, dos Santos CB, Ribeiro RPP. Educação alimentar na obesidade: Adesão e resultados antropométricos. Revista de Nutrição. 2011; 24(4): 575-584.

15. Neufeld CB, Affonso G, Moreno AL. PRO-META: Programa Cognitivo-Comprotamental de Educação Alimentar em Grupo. In: Neufeld C. Intervenções e pesquisa em terapia cognitivo-comportamental com indivíduos e grupos. Nova Hamburgo: Synopsys; 2014; 44-80.

16. World Health Organization (WHO) [Homepage na internet] Obesity. [acesso em 27, setembro, 2017]. Disponível em: http://www.who.int/topics/obesity/ en/

17. Cunha JÁ. Manual da versão em português das Escalas Beck. São Paulo: Casa do Psicólogo, 2001.

18. Beck AT, Steer RA. Beck Depression Inventory. San Antonio, TX: Psichological Corporation; 1993b.

19. Beck AT, Steer RA. Beck Anxiety Inventory. San Antonio, TX: Psichological Corporation; 1993a.

20. Beck AT, Steer RA. Beck Hopelessness Scale. San Antonio, TX: Psichological Corporation; 1993c.

21. Freitas S, Lopes CS, Coutinho W, Appolinario JC. Tradução e adaptação para o português da Escala de Compulsão Alimentar Periódica. Revista brasileira de psiquiatria. 2001; 23(4): 215-220.

22. Kakeshita IS, Silva AIP, Zanatta DP, Almeida SS. Construção e fidedignidade teste-reteste de escalas de silhuetas brasileiras para adultos e crianças. Psicologia: Teoria e Pesquisa. 2009; 25(2): 263-270.

23. Matos MIR, Aranha LS, Faria AN, Ferreira SRG, Bacaltchuck J, Zanella MT. Binge eating disorder, anxiety, depression and body image in grade III obese patients. Revista Brasileira de Psiquiatria. 2002; 24(4): 165-169.

24. Soares ASB. Qualidade de vida, depressão e ansiedade em indivíduos que procuram tratamento para a obesidade [Dissertação de Mestrado]. Porto: Universidade Fernando Pessoa; 2009.

25. Tosetto AP, Simeão Júnior CA. Obesidade e sintomas de depressão, ansiedade e desesperança em mulheres sedentárias e não sedentárias. Medicina (Ribeirão Preto). 2008; 41 (4): 497-507.

26. Silva I, Pais-Ribeiro J, Cardoso H. Cirurgia de Obesidade: qualidade de vida e variáveis psicológicas antes e depois do tratamento cirúrgico. Psicologia: teoria e prática. 2009; 11(2): 196-210.

27. Oliveira, APDSV, da Silva, MM. Fatores que dificultam a perda de peso em mulheres obesas de graus I e II. Revista Psicologia e Saúde. 2014; 6(1): 74-82.

28. Porto MCV, Brito IC, Calfa ADF, Amoras M, Villela NB, Araújo LMB. Perfil do obeso classe III do ambulatório de obesidade de um hospital universitário de Salvador, Bahia. Arquivos Brasileiros de Endocrinologia e Metabolismo. 2006; 46(6): 668-673.

29. Gigante DP, Dias-da-Costa JS, Olinto MTA, Menezes AMB, Macedo S. Obesidade da população adulta de Pelotas, Rio Grande do Sul, Brasil e associação com nível socioeconômico. Cadernos de Saúde Pública. 2006; 22(9): 1873-1879.

30. Sarturi JB, Neves J, Peres KG. Obesidade em adultos: estudo de base populacional num município de pequeno porte no sul do Brasil em 2005. Ciência e Saúde Coletiva. 2010; 15 (1): 105-113.

31. Dong C, Sanchez LE, Price RA. Relationship of obesity to depression: a family-based study. International Journal of Obesity. 2004; 28(6): 790-795.

32. Baptista MN, Vargas JF, Baptista ASD. Depressão e qualidade de vida em uma amostra brasileira de obesos mórbidos. Avaliação Psicológica. 2008; 7(2): 235247.

33. Saueressig MR, Berleze KJ. Análise de influências em dietas de emagrecimento. Psico. 2009; 41 (2): 231238.

34. Duchesne, M, Almeida, PEDM. Cognitive-behavioural therapy of eating disorders. Revista Brasileira de Psi- 
quiatria. 2002; 24: 49-53.

35. Manfro, GG, Heldt, E, Cordioli, AV, \& Otto, MW. Cognitive-behavioral therapy in panic disorder. Revista Brasileira de Psiquiatria. 2008; 30: 81-87.

36. Silvestri E, Stavile A. Aspectos Psicológicos de la obesidad: Importancia de su identificación y abordaje dentro de un enfoque interdisciplinario [Tese de Doutorado] Córdoba: Universidad Autónoma de Favaloro; 2005.

37. Reyes, AN, Fermann, IL. Eficácia da terapia cognitivo-comportamental no transtorno de ansiedade generalizada. Revista Brasileira de Terapias Cognitivas. 2018; 13(1): 49-54.

Recebido em: 08/10/2018

Aceito em: 21/05/2019 